\title{
Supportive treatment of vascular dysfunction in pediatric subjects with obesity: the OBELIX study
}

\author{
Luca Pecoraro ${ }^{1,2,7}$, Thomas Zoller ${ }^{3,7}$, Richard L. Atkinson ${ }^{4}$, Fulvio Nisi ${ }^{5}$, Franco Antoniazzi ${ }^{3}$, Paolo Cavarzere ${ }^{3}$, Giorgio Piacentini ${ }^{3}$ and $^{3}$ \\ Angelo Pietrobelli iD ${ }^{3,6}$ 凶
}

(c) The Author(s) 2022, corrected publication 2022

\begin{abstract}
INTRODUCTION: Overweight or obese children develop abnormal endothelial cell dysfunction and arterial intima-media thickening with increased vasomotor tone and inflammation. Curcumin, resveratrol, zinc, magnesium, selenium, and vitamin D have shown beneficial effects on endothelial function. We test, among overweight and obese pediatric subjects, the effects on the endothelium of a combination of curcumin, resveratrol, zinc, magnesium, selenium, and vitamin D.

METHODS: Forty-eight subjects (6-17 years) were randomized into two groups (placebo vs treatment) attended three visits at 0, 3, and 6 months ( \pm 15 days). Endothelial function was assessed by means of a post-occlusive release hyperemic (PORH) test for estimation of delta flow (DF) and hyperemic AUC index, and a heat provocation test (HPT) to measure DF HPT (DF RESULTS: Significant DF difference was noted at 6 months in both groups $(p<0.001)$. Overall time trend was significantly different between baseline, 3 months, and 6 months both in placebo $(p<0.05)$ and treatment $(p<0.001)$ groups and their comparison $(p<0.001)$. No differences were noted in hyperemic AUC index ( 3 and 6 months), whilst there were significant differences in time trends of rreatment $(p<0.001)$ and placebo $(p<0.05)$ groups and their comparison $(p<0.001)$. DF HPT difference between groups was significant at 3 and 6 months $(p<0.05)$. The overall time trend was significant exclusively in Treatment group between 3 and 6 months $(p<0.05)$. Correlation with anthropometrics was found for DF and body mass index $(r=0.6776$ months, $p<0.05)$, as well

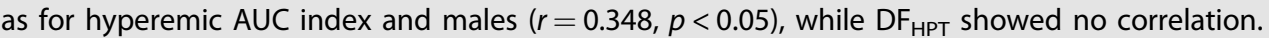

CONCLUSION: Curcumin, resveratrol, zinc, magnesium, selenium, and vitamin D appear to be promising in enhancing endothelial function by improvement of both DF in the PORH test and DF in the HPT, lowering the risk of developing cardiovascular diseases in overweight and obese pediatric subjects.
\end{abstract}

Nutrition and Diabetes (2022)12:2 ; https://doi.org/10.1038/s41387-021-00180-1

\section{INTRODUCTION}

Pediatric overweight and obesity are traditionally characterized by an excess of body fat [1], which is an independent cardiovascular risk factor that could lead to type 2 diabetes, hypertension, insulin resistance, and reduced endothelial function development [2]. The link between excess body fat, endothelial dysfunction, and insulin resistance is related to the fact that endothelium-dependent vasodilatation is impaired in proportion to insulin resistance and other adiposity-related indices [3].

Bussey and colleagues in 2016 found reduced nitric oxide (NO) production and increased inflammation in perivascular adipose tissue (PVAT) of obese mice compared to nonobese controls. The same study found significantly improved PVAT anticontractile function after weight loss by reduced adipose inflammation and increased NOS availability [4].

Similar results were previously stated by Ketonen et al. in 2010, they found impaired endothelium-dependent vasodilation in response to acetylcholine in obese mice receiving high-fat diet in comparison with mice receiving a normal fat diet. Differences between the obese and control groups markedly reduced after the introduction of caloric restriction in the obese group [5].

Moreover, it is very well known that adipose tissue is a key regulator of inflammation with the secretion of proinflammatory cytokines (i.e., adipokines) that play a role in influencing glucose metabolism and endothelial function [6]. Children who are overweight or obese develop abnormal endothelial cell dysfunction and arterial intima-media thickening with increased vasomotor tone and inflammation [7, 8]. This may lead to atherosclerotic plaques formation [9]. The endothelium contributes to blood pressure and flow regulation by releasing nitric oxide (NO) and other compounds, which contribute either to vasodilation or vasoconstriction $[10,11]$. On the other hand, the interaction between endothelium and adipokines suggests a role for adipokines in vascular homeostasis and ultimately in the mechanisms for the development of cardiovascular diseases [12-14]. Also, a healthy endothelium prevents platelet aggregation, proliferation of vascular smooth muscle cells, adhesion, and subsequent diapedesis of leukocytes through the vascular wall [15]. The endothelium plays a unique role in vascular homeostasis

\footnotetext{
${ }^{1}$ Department of Medicine, University of Verona, Verona, Italy. ${ }^{2}$ Paediatric Clinic, ASST Mantua, Mantua, Italy. ${ }^{3}$ Pediatric Unit, Department of Surgical Sciences, Dentistry, Gynecology and Pediatrics, University of Verona, Verona, Italy. ${ }^{4}$ Department of Medicine, Virginia Commonwealth University, Richmond, VA, USA. ${ }^{5}$ Humanitas Clinical and Research Center-IRCCS,
}

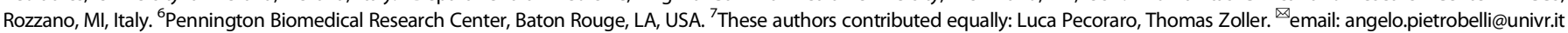


Table 1. Baseline Characterisitics of the study population.

\begin{tabular}{|c|c|c|}
\hline & $N$ & $\%$ \\
\hline Patients enrolled & 48 & \\
\hline Drop-outs & 21 & 43.75 \\
\hline Age (years) & $12.85 \pm 3.04$ & \\
\hline Gender (male) & 25 & 52.08 \\
\hline Weight (kg) & 76.90 & [66.0-92.5] \\
\hline BMI & $30.89 \pm 5.22$ & \\
\hline BMI Z-score & 0.61 & [0.22-1.12] \\
\hline \multicolumn{3}{|l|}{ Ethnicity } \\
\hline Caucasic & 41 & 85.42 \\
\hline North African & 4 & 8.33 \\
\hline Moroccan & 3 & 6.25 \\
\hline \multicolumn{3}{|l|}{ Sport } \\
\hline Sport type score ${ }^{a}$ & 3 & {$[2-3]$} \\
\hline hours/week & 3 & [1-4] \\
\hline \multicolumn{3}{|l|}{ Dietary fat intake } \\
\hline Low & 23 & 47.92 \\
\hline Medium & 23 & 47.92 \\
\hline High & 2 & 4.17 \\
\hline \multicolumn{3}{|l|}{ Drugs } \\
\hline D-Vitamin & 8 & 16.67 \\
\hline Methylphenidate & 1 & 2.08 \\
\hline Montelukast & 1 & 2.08 \\
\hline None & 38 & 79.17 \\
\hline
\end{tabular}

Data are expressed as number $(N)$ and percentage or mean \pm SD or median [IQR] as appropriate.

${ }^{\text {a }}$ Sport type $=($ dynamic score $) \times($ static score $)$.

that is maintained by endothelium-derived biomolecules with different functions (i.e., vasodilation, vasoconstriction, growth promoter, growth inhibitor, adhesion molecules, thrombolytic factors) [15]. Endothelium-dependent damage arises from metabolic abnormalities of glucose metabolism that lead to vascular dysfunction [15]. Endothelial dysfunction characterized by abnormal vasodilator response and increased arterial stiffness is associated with an increased risk of cardiovascular events [15] and is present in pediatric subjects with obesity [11]. The major goal of obesity therapy in children should be the reduction of the long-term risks of cardiovascular diseases. Since damaged endothelium is so involved in the development of later risks of morbidity and mortality, it may be useful to monitor and eventually treat endothelium status in order to prevent longterm risk factors [16-20]. Among different therapies, curcumin showed promising results in inhibition of advanced glycation end product-induced oxidative stress and inflammatory responses in endothelial cell damage [14]. Curcumin is the most active component of the curcuminoids extracted from Curcuma longa $L$., and it has been demonstrated to protect against cellular inflammatory responses and oxidative stress in vascular complications as well as endothelial damage $[16,17]$. Another antioxidant, resveratrol, has shown beneficial effects on endothelial function since it has the ability to increase NO synthesis that, in vivo, plays an antioxidant function in the endothelium [18-20]. Animal models showed a relationship between prenatal and neonatal zinc deficiency and vascular dysfunction $[21,22]$ keeping in mind that zinc has antioxidant-like properties in activated endothelium cells [23]. Magnesium, an essential mineral for human health, plays a role in endothelium function and participates in vascular calcification [24]. Magnesium supplementation revealed a significant improvement of flow-mediated dilation and pulse wave velocity [25]. Recent findings showed the active role of selenium in endothelial function [24]. Specifically, selenium and seleno protein are associated with endothelial cytoprotection [24], having a role on endothelium activation biomarkers [26]. Among several functions, vitamin $D$ is associated with endothelial dysfunction [27] showing anti-inflammatory effects through suppression of tumor necrosis factor-a and the release of interleukin-6 [28].

To the best of our knowledge, we did not find information regarding studies done in pediatrics, looking at endothelium dysfunction treatment. In light of these findings, using a doubleblind randomized control study with a rigorous approach, we tested the effects on the endothelium of a combination of Curcumin, resveratrol, plus zinc, magnesium, selenium, and vitamin $\mathrm{D}$ in a cohort of pediatric subjects with obesity.

\section{METHODS}

\section{Participants selection}

In this study, we recruited 48 children aged 6-17 years who were obese as defined by a body mass index (BMI) higher than the 95 percentile for age based on the Centers for Disease Control and Prevention standard [29]. Children with genetic syndromes or cardiovascular diseases were excluded from the study. The study was approved by the local Ethical Committee (OBELIX: code CE 5384, 2019). Informed written consent for study participation was collected from legal caregivers of each participant and from participants older than 10 years during the first visit. Participants were asked to attend three visits at 0,3 , and 6 months ( \pm 15 days).

\section{Randomization}

This study was a double-blind randomized control study done with a rigorous approach. Using a computer-generated randomization schedule, study supplement and placebo were randomized (1:1) into 70 batches (each consisting of 6 packs containing 30 tablets a pack) and each was given a unique identification number. The coordinator of the study maintained the randomization list. Study physicians, other study personnel, and parents or legal guardians were blinded to the batches of medication and to the identification. Subjects who satisfied the inclusion for the study were assigned an identification number (linked to a batch) in sequential order. Since the randomization list was made before the batch assignment and later preserved in a closed envelope that made it unavailable for the entire duration of the study, neither study physicians nor patients could know subjects belonging to Placebo or Treatment group.

Subjects took one tablet per day orally starting day 1 after the visit and continuing for the 6-month duration of the study. A number of tablets not taken $\geq 2$ tablets per month were considered not adherence to the study (drop out).

\section{Supplements characteristics}

Treatment tablet composition (Auxilie ${ }^{\circledR}$ Immuplus, Envicon Medical, Verona, Italy): vitamin D3: $25.00 \mu \mathrm{g}$, folic acid: $90.00 \mu \mathrm{g}$, selenium: $55.00 \mu \mathrm{g}$ magnesium: $300.00 \mathrm{mg}$, zinc: $7.00 \mathrm{mg}$, curcum (Meriva ${ }^{\oplus}$ ): $100.00 \mathrm{mg}$, Polygonum dry extract: $20 ., 41 \mathrm{mg}$ (of which resveratrol: $20.00 \mathrm{mg}$ ), Soy dry extract: $37.50 \mathrm{mg}$.

Placebo tablet composition: saccharose, fructose, aroma, antiagglomerate agents: fatty acids, magnesium salts, silicium dioxide, colorant: riboflavin 5-sodium phosphate; sweetener: stevia glycoside, sucralose, neohesperidin DC.

Both tablets (treatment and placebo) were similar in form, color, and flavor.

\section{Anthropometric measurements and habits}

Height $(\mathrm{cm})$ and weight $(\mathrm{kg})$ were measured for each child at every visit. $\operatorname{BMI}\left(\mathrm{kg} / \mathrm{m}^{2}\right)$ was calculated as raw value and as $Z$-score for age. At the first visit, we conducted an oral interview with both parents and children and we collected information regarding sport/exercise practices and dietary habits.

\section{Endothelial function}

Endothelial function was assessed using two methodologies: a postocclusive release hyperemic test (PORH) and a heat provocation test (HPT). 
Table 2. Characteristics of completers: Placebo vs Treatment groups.

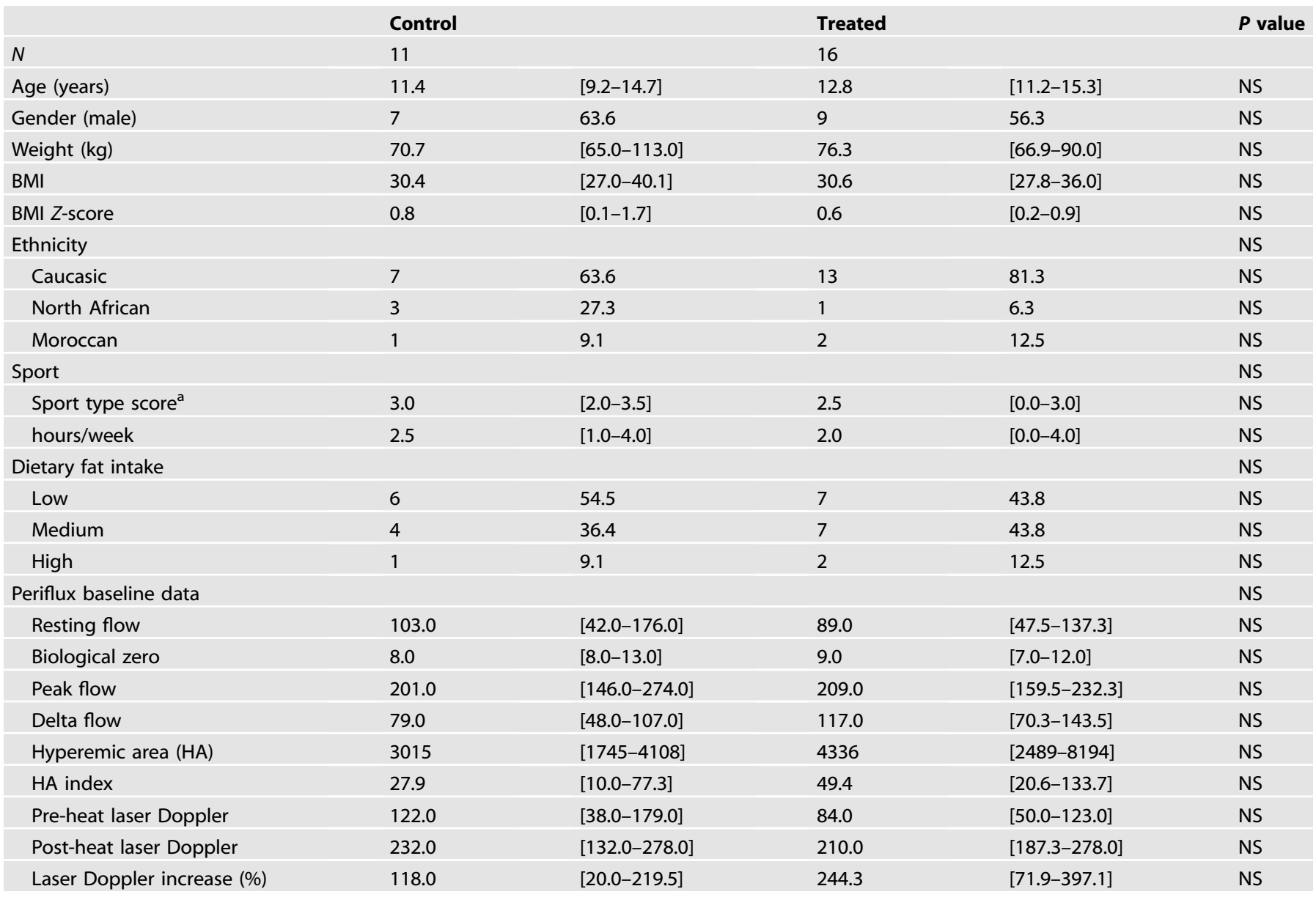

Data are expressed as median [IQR] or number and percentage as appropriate.

AUC area under the curve, HPT heat provocation test, NS not significant.

${ }^{\text {a }}$ Sport type $=($ dynamic score $) \times($ static score $)$

Subjects were laid on a bed with the upper extremity positioned at $45^{\circ}$ and a cuff was placed around the medium third of the forearm in order to occlude the radial and ulnar arteries. The probe was positioned over the volar aspect of the hand at the first finger distal metacarpal surface and the hand was gently immobilized in order to minimize the occurrence of motion artefacts. All tests were performed with a laser Doppler sensor (Periflux 6000 System integrated with a thermostatic 457 probe, Perimed, Sweden).

Post-occlusive release hyperemia. Once cutaneous blood flow over the area became stable, basal values were recorded for 2 min and then the pressure within an inflatable cuff placed at the forearm and connected to a computer-controlled manometer was raised to $200 \mathrm{~mm} \mathrm{Hg}$ for $3 \mathrm{~min}$. Using a computer-controlled pressure release to allow for consistent deflation times, the cuff was rapidly deflated and the laser Doppler measured hyperemic responses over the next $2 \mathrm{~min}$. Commercially available software (Perimed, Järfälla, Sweden) allowed for unbiased estimates of DF, hyperemic AUC and hyperemic AUC index.

Delta flow. The flow variation from resting flow to peak flow (maximal arterial flow achieved after abrupt cessation of occlusion) is called DF. DF was computed at baseline, after 3 months and after 6 months in each group. DF was compared between the two groups and its temporal trend was evaluated.

Hyperemic AUC and hyperemic AUC index. Hyperemic AUC is the difference between the area under the hyperemia zone and the area under the rest flow zone expressed in perfusion units multiplied by time.
We also chose to compute an index named "hyperemic AUC index" in order to better reflect the real value of $A U C$ in relation to the resting flow of each participant. AUC index $=$ AUC/RF.

Heat provocation test. After the PORH test, we wait at least 2 min to proceed with the HPT in order to re-establish basal blood flow under the probe. The HPT consisted of recording resting blood flow in the forearm for $2 \mathrm{~min}$, heating the forearm by raising the temperature of the probe to $44^{\circ} \mathrm{C}$ and recording the hyperemic response induced by the heat expressed as a percentage difference in perfusion units above resting flow. This was defined as DF $_{\mathrm{HPT}}$.

\section{Statistical analysis}

Qualitative variables were expressed as percentages and 95\% confidence intervals $(95 \% \mathrm{Cl})$, and quantitative variables as means SD or medians and interquartile ranges depending on whether the variables were normally distributed. The Kolmogorov-Smirnov test was used to ascertain the normal distribution. Student's $t$ test and analysis of variance or Mann-Whitney test, Wilcoxon's signed-rank test and Kruskal-Wallis test were used as appropriate to the data to compare distributions of DF,

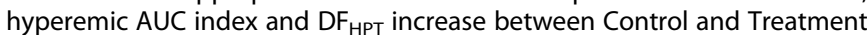
groups at fixed time intervals (0, 3 and 6 months) and to evaluate the trend of changes over time within each group. The association between each of these Periflux parameters at baseline $(T 0)$ and some relevant population characteristics such as BMI, gender, ethnicity, dietary fat intake, sport type and hours per week was tested using Pearson's $r$ correlation coefficient or Spearman's correlation coefficient as appropriate. MedCalc Statistical Software version 17.6 (MedCalc Software bvba, Ostend, Belgium; http:// 
www.medcalc.org) and GraphPad Prism version 6.00 for Mac (GraphPad Software, La Jolla California USA, http://www.graphpad.com) were used to perform the analyses and $a$ was set at 0.05 .

\section{RESULTS}

Forty-eight subjects were recruited and submitted to a questionnaire, medical examination, anthropometric evaluation and measurement of endothelial function. Twenty-one subjects dropped out. Nine subjects were not compliant with tablet prescription (two or more tablets not taken each month), whilst 12 patients did not attend 3- and 6-month follow-up due to personal reasons (mainly young subjects who found the 3 min cuff occlusion too annoying to tolerate).

Among the patients who completed the study, 16 patients took antioxidant supplementation and 11 took placebo. No one reported adverse effects.

The characteristics of all subjects at baseline are shown in Table 1. The characteristics and homogeneity of the subjects who completed the study are shown in Table 2. "Treatment group" and "Placebo group" were compared at baseline and were homogeneous with no significant difference in anthropometric and endothelial function parameters.

\section{Delta flow}

"Treatment group" and "Placebo group" did not show differences in PORH DF at baseline and 3 months (Table 3). On the other hand, a significant difference was present at 6 months (78.5 [72.0-94.0] vs 63.0 [53.0-78.0], respectively, treatment and control, $p<0.001$ ). Overall time trend of "Treatment group" and "Placebo group" was detected and compared with Kruskal-Wallis test, showing a significant difference in the flow time trend between baseline, 3 months, and 6 months both in the "Placebo group" $(p<0.05)$ and "Treatment group" $(p<0.001)$ (Fig. 1). Moreover, the comparison between the two groups was highly significant $(p<0.001)$. Regarding the correlation between anamnestic-anthropometric data and endothelial function parameters at baseline, the flow variation from resting flow to peak flow (DF) showed a significant correlation with BMI (Spearman's $r=0.355, p<0.05$ ). The strength of this correlation increases with time; specifically, the correlation between BMI and DF was stronger at 6 months ( $r=0.677)$ (Fig. 2 and Table 4).

\section{Hyperemic AUC index}

"Treatment group" and "Placebo group" did not show mutual differences in "hyperemic AUC index" at 3 and 6 months (Table 3). An overall time trend of "Treatment group" and "Placebo group" was detected and compared with Kruskal-Wallis test, showing a significant difference in the Hyperemic AUC index both in the "Placebo group" ( $p<0.05)$ and "Treatment group" $(p<0.001)$ (Fig. 1). In addition, the comparison between the time trends of the two groups was very significant $(p<0.001)$. About the relationship between anamnestic and anthropometric data and endothelial function parameters at baseline, the hyperemic AUC index showed a significant correlation with the male gender (Spearman's $r=0.348$, $p<0.05$ ) (Table 4).

\section{Delta flow heat provocation test}

"Treatment group" and "Placebo group" showed a significant difference between groups in DF $_{\mathrm{HPT}}$ when comparing the results of the "HPT" at 3 months and 6 months $(p<0.05)$ (Table 3$)$. The overall time trend of the "Treatment group" and "Placebo group" was detected and compared with Kruskal-Wallis test. In the "Placebo group," there was no significant difference in the DF analysis between baseline, 3 months, and 6 months. In the

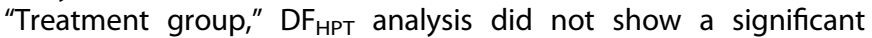
difference between baseline and 3 months. However, there was a significant difference between 3 and 6 months $(p<0.05)$ in this 

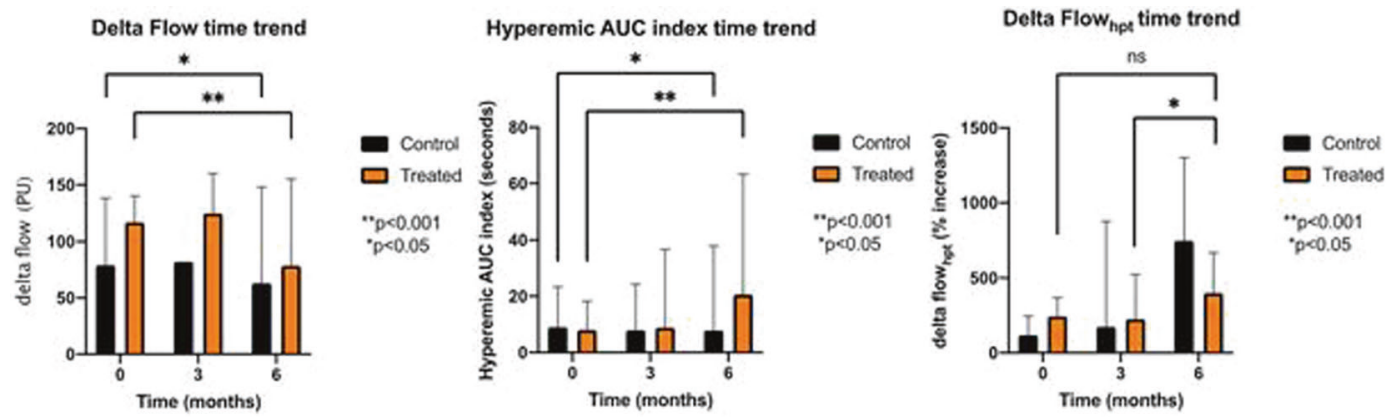

Fig. 1 Time trend variation of Periflux data. Difference in the flow time trend between baseline, time-3-months, and time-6-months both in the placebo group and in the treatment group. ${ }^{*} p<0.05 .{ }^{* *} p<0.001$. ns not significant, PU perfusion unit, AUC area under the curve, HPT heat provocation test.

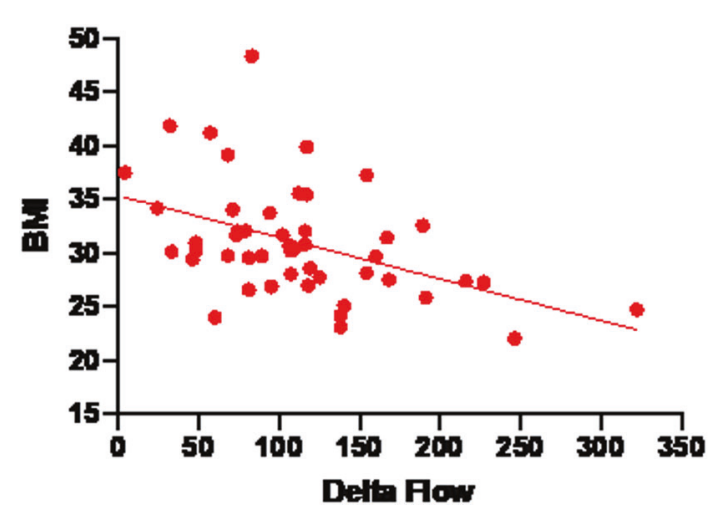

Fig. 2 Correlation of delta flow and body mass index (BMI). This correlation increases with time and is stronger at time-6-months $(r=0.677)$.

group (Fig. 1). Regarding the association between anamnestic and anthropometric data and endothelial function parameters at baseline, $\mathrm{DF}_{\mathrm{HPT}}$ did not show a significant correlation with anamnestic and anthropometric parameters (Table 4).

\section{DISCUSSION}

The results of the study illustrate the correlation between obesity status and endothelial dysfunction in children, showing that cardiovascular damages begin early in life. Although the instrument and the methodology in use in this study are considerably new, we used the most validated parameters such as DF, hyperemic area under the curve and $D_{\mathrm{HPT}}$, to estimate endothelial function or dysfunction in adults [30]. Previous studies showed that endothelial dysfunction was associated with adiposity in obese children as well $[6,31,32]$.

Our results showed that DF improved significantly $(p<0.001)$ in the Treatment group when compared with the Control group. It should be noted that all the enrolled subjects, both treatment and controls, have had some benefits in their endothelial function over time, perhaps due to the fact that some educational advice was given during the medical assessment. However, in the Treatment group, this benefit was constantly and significantly higher $(p<$ 0.001 ), implying a role of the supplements in promoting a higher degree of improvement. The correlation between basal DF and basal BMI shown in Fig. 1 is negative, indicating that DF decreases while BMI increases, once again, underlining the treatment effect in enhancing endothelial performances despite an increase in the BMI.

Although we did not find any correlation with dietary fat content, type, and hours spent on physical activities, sports, or ethnicity, we may speculate that a larger sample size and/or different ethnicity could show significant results. It is important to recognize that our population was mostly Caucasian. Regarding physical activity, previous studies showed improvement of arterial stiffness, reduction of abdominal fat, increases cardiorespiratory fitness, and delayed arterial wall remodeling in prepubertal obese children [31].

We did not find any significant correlation between groups in the area of hyperemia, showing a similar trend of time variation in both groups. The influence of advice by the physician in promoting changes in both groups cannot be excluded. However, the greater changes in the treated group in comparison with the control group suggests a treatment effect of the supplements in modifying the "hyperemic area under the curve." Again, a larger sample size or an even longer observational/treatment period might influence future results.

Regarding gender, males showed a wider range of hyperemia in comparison with females and despite the small sample size, North African subjects showed a higher range of hyperemia, followed by Italo-Moroccan and Caucasians. In contrast with our results, Mueller et al. [33] found that in females endothelium function was lower than in men. However, the cohort of adolescents in the study of Mueller et al. [33] was mainly healthy. Adult men usually develop cardiovascular diseases at a younger age and have a higher propensity to develop coronary heart diseases than women [34].

Looking at the "DH provocation test," significant changes between the treated group and control group were found after 3 months of treatment $(p<0.05)$, giving us the impression that the combination of Curcumin and resveratrol plus zinc, magnesium, selenium, and vitamin $D$ require some time to influence endothelium function per se.

Regarding the relationship between endothelial function and $\mathrm{BMI}$, a recent systematic review and meta-analysis in adults showed that diet improved endothelium function independently from BMI [35], but we did not find similar results in children using the same approach and measurements.

In summary, Curcumin, resveratrol, zinc, magnesium, selenium, and vitamin D appear to be promising in enhancing endothelial function by improvement of both DF in the PORH test and DF in the heat provocation test, although it seems the combination effectively acts at least after 3 months since the therapy started.

The primary mechanism of action of polyphenols was originally thought to lie in their direct antioxidant effects. However, a number of other possible biochemical and molecular mechanisms have been identified, including multifarious effects within intraand intercellular signaling pathways that govern antioxidative properties like nuclear factor E2-related factor 2 and inflammation pathways, e.g., nuclear factor- $\mathrm{KB}$ and thus modulating the synthesis of inflammatory mediators including cytokines tumor

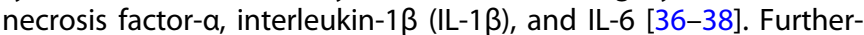
more, Curcumin and resveratrol have been demonstrated to exert 
epigenetic regulatory roles including the inhibition of DNA methyltransferases, regulation of histone modifications via the regulation of histone acetyltransferases and histone deacetylases, regulation of microRNAs, and action as a DNA-binding agent $[38,39]$. The accumulation of visceral fat in obesity is associated with a state of chronic oxidative stress and excessive production of proinflammatory adipokines, which contributes to a low-grade chronic inflammation state that can be attenuated with magnesium [40], zinc [41], and selenium supplementation as recognized antioxidant trace elements [42]. The effects we observed have biological plausibility. Daily vegetable consumption was associated with more favorable arterial function [43] and the same was observed increasing the polyphenol content of the diet via consumption of fruit and vegetables [44], with a dose-dependent effect [45]. Red wine consumption has been shown to positively influence processes involved in vascular dysfunction [46] and resveratrol, one of the main compounds of the tablets used in our study, is the major polyphenol in wine. A systematic review and meta-analysis of randomized controlled trials clearly documented that resveratrol [47] or curcumin [48] intervention significantly increased flow-mediated dilatation. Several studies documented that a high intake of dietary polyphenols inhibit endothelial dysfunction and induce vascular endothelium-dependent vascular relaxation viz. redox regulation and NO production [47] and may have a preventive effect against cardiovascular diseases $[49,50]$. Moreover, vitamin D insufficiency is associated with increased arterial stiffness and endothelial dysfunction [51], and vitamin D levels are inversely associated with increased arterial stiffness in a normative aging population, irrespective of the traditional risk factor burden [52]. Its supplementation improved NO-dependent arteriolar vasodilation in obese adults [53] and is associated with decreased vascular dysfunction in patients with chronic kidney disease [54]. Hard water consumption seems to be protective against cardiovascular diseases particularly in relation to its magnesium content [55] and a meta-analysis suggest that oral Mg supplementation may improve endothelial function when conducted at least for 6 months and in unhealthy, overweight or older individuals [56] as observed in our studied children. In animal models, zinc deficiency is associated with reduced vasodilator response [21], and in humans, zinc supplementation alleviates diabetic endothelial dysfunction [22]. Zinc is a protective and critical nutrient for the maintenance of endothelial integrity [23] through attenuation of tumor necrosis factor-mediated activation of upregulation of inflammatory cytokines in endothelial cells [57]. In rats, it has been documented that hypercholesterolemia, a condition commonly found in obese subjects, promotes endothelial dysfunction in the presence of selenium deficiency [58] and many studies in humans indicate an association between selenium deficiency and increased risk of morbidity and mortality [24]. Selenium supplementation has been shown to significantly reduce the risk for cardiovascular mortality in patients with diabetes, hypertension, and ischemic heart disease [59].

According to the study results, looking at the day-by-day clinical work together with regular physical activity, an increased consumption of the correct diet containing nutritive and nonnutritive compounds may contribute to the improvement of the quality of life by delaying the development of endothelial dysfunction in pediatric subjects with obesity.

Despite intriguing findings, our study has some limitations. The high drop-out level we had in our study population is in line with the low adherence of obese children to health-behavior recommendations [60] and this fact may also be responsible for the partial effect we observed with the nutraceutical supplementation. Moreover, the potential effect of growth on our subjects has not been taken into consideration in the study.

It is well known that the laser Doppler technique used to evaluate the endothelial function and ultimately cardiovascular risk in adults is used only in research and no clinical studies can be found. We did not 
find any guideline or protocol to diagnose endothelial dysfunction using this technique. Another important point that needs to be taken into consideration is the time during the day of the vascular assessment. We know that fasting and "circadiam rhythm" may influence results per se and we did not have the possibility to take measurements at the same time of the day, although we tried very hard to standardize the time of measurements.

Treatment of obesity is always an issue and since there is a high prevalence of inflammation in obese subjects leading to several problems including endothelial dysfunction and related cardiovascular complications [61], substances with antioxidant, antiinflammatory, angiogenic, and platelet aggregation properties such as resveratrol and curcumin, zinc, selenium, magnesium, folic acid, and vitamin $D[16,61,62]$ may have protective effects to improve endothelial dysfunction, thus lowering the risk of developing cardiovascular diseases [63]. In conclusion, it is possible to speculate that multicomponent supplementation with minerals, vitamin $D$, and functional food-derived factor that resembles but not a substitute for a healthy diet may help to improve vascular dysfunction in obese children [8].

\section{REFERENCES}

1. Greydanus DE, Agana M, Kamboj MK, Shebrain S, Soares N, Eke R. et al. Pediatric obesity: current concepts. Dis Mon. 2018;64:98-156.

2. De Meneck F, Victorino de Souza L, Oliveira V, do Franco MC. High irisin levels in overweight/obese children and its positive correlation with metabolic profile, blood pressure, and endothelial progenitor cells. Nutr Metab Cardiovasc Dis. 2018;28:756-64.

3. Avogaro A, de Kreutzenberg SV. Mechanism of endothelial dysfunction in obesity. Clin Chim Acta. 2005;360:9-26.

4. Bussey CE, Withers SB, Aldous RG, Edwards G, Heagerty AM. Obesity-related perivascular adipose tissue damage is reversed by sustained weight loss in the rat. Arterioscler Thromb Vasc Biol. 2016;36:1377-85. https://doi.org/10.1161/ ATVBAHA.116.307210.

5. Ketonen J, Pilvi T, Mervaala E. Caloric restriction reverses high-fat diet-induced endothelial dysfunction and vascular superoxide production in C57BI/6 mice. Heart Vessels. 2010;25:254-62. https://doi.org/10.1007/s00380-009-1182-x.

6. Montero D, Walther G, Perez-Martin A, Roche E, Vinet A. Endothelial dysfunction, inflammation, and oxidative stress in obese children and adolescents: markers and effect of lifestyle intervention. Obes Rev. 2012;13:441-55.

7. Ciccone MM, Miniello V, Marchioli R, Scicchitano P, Cortese F, Palumbo V, et al. Morphological and functional vascular changes induced by childhood obesity. Eur J Cardiovasc Prev Rehabil. 2011;18:831-35.

8. Bruyndonckx L, Hoymans VY, Lemmens K, Ramet J, Vrints CJ. Childhood obesityrelated endothelial dysfunction: an update on pathophysiological mechanisms and diagnostic advancements. Pediatr Res. 2016;79:831-37.

9. Kim SH, Despres JP, Koh KK. Obesity and cardiovascular disease: friend or foe? Eur Heart J. 2016;37:3560-8.

10. Sorop O, Olver TD, van de Woow J, Heinnonen I, van Doin RW, Duncker DJ, et al. The microcirculation: a key player in obesity-associated cardiovascular disease. Cardiovasc Res. 2017; 113:1035-45.

11. Sowka A, Dobrzyn P. Role of perivascular adipose tissue-derived adiponectin in vascular homeostasis. Cells. 2021;10:1485.

12. Schinzari F, Tesauro M, Cardillo C. Endothelial and perivascular adipose tissue abnormalities in obesity-related vascular dysfunction. Novel targets for treatment. J Cardiovasc Pharmacol. 2017;69:360-68.

13. Vanhoutte PM, Shimokawa H, Feletou M, Tang EH. Endothelial dysfunction and vascular disease: a 30th anniversary update. Acta Physiol. 2017;219:22-96.

14. Kheirandish-Gozal L, Bhattacharjee R, Kim J, Clair HB, Gozal D. Endothelial progenitor cells and vascular dysfunction in children with obstructive sleep apnea. Am J Respir Crit Care Med. 2010;182:92-97.

15. Bruyndonckh L, Hoymans VY, Van Cranenbroeck AH. Assessment of endothelial dysfunction in childhood obesity and crilical use. Oxid Med Cell Longev. 2013;2013:1-19.

16. Sun YP, Gu JF, Tan XB, Wang CF, Jia XB, Feng $L$, et al. Curcumin inhibits advanced glycation end product-induced oxidative stress and inflammatory responses in endothelial cell damage via trapping methylglyoxal. Mol Med Rep. 2016;13:1475-86

17. Kocaadem B, Sanlier N. Curcumin, an active component of turmeric (Curcuma longa), and its effects on health. Crit Rev Food Sci Nutr. 2017;57:2889-95.

18. Van der Spy WJ, Pretorius E. Is the use of resveratrol in the treatment and prevention of obesity premature? Nutr Res Rev. 2009;22:111-17.
19. Das KD, Maulik N. Resveratrol in cardioprotection: a therapeutic promise of alternative medicine. Mol Interv. 2006;6:36-47.

20. Xia N, Daiber A, Förstermann U, Li H. Antioxidant effects of resveratrol in the cardiovascular system. Br J Pharmacol. 2017;174:1633-46.

21. Mendes Garrido Abregú F, Gobetto MN, Juriol LV, Caniffi C, Elesgaray R, Tomat AL, et al. Developmental programming of vascular dysfunction by prenatal and postnatal zinc deficiency in male and female rats. J Nutr Biochem. 2018;56:89-98.

22. Liu P, Liu J, Wu Y, Xi W, Wei Y, Yuan Z, et al. Zinc supplementation protects against diabetic endothelial dysfunction via GTP cyclohydrolase 1 restoration. Biochem Biophys Res Commun. 2020;521:1049-54.

23. Hennig B, Meerarani P, Toborek M, McClain CJ. Antioxidant-like properties of zinc in activated endothelial cells. J Am Coll Nutr. 1999;18:152-8.

24. Lopez Juonor E, Leite HP, Konstantyner T. Selenium and selenoproteins: from endothelial cytoprotection to clinical outcomes. Trans Res. 2019;208:85-104.

25. Marquez BCAA, Klein MRST, Rabello de Cunha M, de Souza Mattos S, de Paula Nogueira $L$, de Paula $T$, et al. Effects of oral magnesium supplementation on vascular function: a systematic review and meta-analysis of randomized controlled trials. High Blood Press Cardiovasc Prev. 2020;27:19-28.

26. Zhang X, Liu C, Guo J, Song Y. Selenium status and cardiovascular diseases: metaanalysis of prospective observational studies and randomized controlled trials. Eur J Clin Nutr. 2016;70:162-69.

27. Kim D-H, Meza CA, Clarke H, Kim J-S, Hickner RC. Vitamin D and endothelial function. Nutrients. 2020;12:575.

28. Ebihara K, Masuhiro $Y$, Kitamoto $T$, Suzawa M, Uematsu $Y$, Yoshizawa $T$, et al. Intron retention generates a novel isoform of the murine vitamin $D$ receptor that acts in a dominant negative way on the vitamin D signaling pathway. Mol Cell Biol. 1996;16:3393-3400.

29. Kuczmarski RJ, Ogden CL, Guo SS, Grummer-Strawn LM, Flegal KM, Mei Z, et al. 2000 CDC growth charts for the United States: methods and development. Vital Health Stat. 2002;11:1-190.

30. Stiefel P, Moreno-Luna R, Vallejo-Vaz AJ, Beltrán LM, Costa A, Gómez L. et al Which parameter is better to define endothelial dysfunction in a test of postocclusive hyperemia measured by laser-Doppler flowmetry?. Coron Artery Dis. 2012;23:57-61.

31. Bruyndonckx L, Hoymans VY, Lemmens K, Ramet J, Vrints CJ. Childhood obesityrelated endothelial dysfunction: an update on pathophysiological mechanisms and diagnostic advancements. Pediatr Res. 2016;79:831-7.

32. Farpour-Lambert NJ, Aggoun Y, Marchand LM, Martin XE, Herrmann FR, Beghetti M. Physical activity reduces systemic blood pressure and improves early markers of atherosclerosis in pre-pubertal obese children. J Am Coll Cardiol. 2009;54:2396-406.

33. Mueller UM, Walther $C$, Adam J, Fikenzer K, Erbs S, Mende M, et al. Endothrlisl function in children and adolescents is mainly influenced by age, sex and physical activity. Circ J. 2017;81:717-25.

34. Bots SH, Peters SAE, Woodward M. Sex differences in coronary heart disease and stroke mortality: a global assessment of the effect of ageing between 1980 and 2010. BMJ Glob Health. 2017;2:2000298.

35. Shannon OM, Mendes I, Köchl C, Mazidi M, Ashor AW, Rubele S, et al. Mediterranean diet increases endothelial function in adults: a systematic review and meta-analysis of randomized controlled trials. Nutrition. 2020;150:1151-9.

36. Kim HS, Quon MJ, Kim AJ. New insights into the mechanisms of polyphenols beyond antioxidant properties; lessons from the green tea polyphenol, epigallocatechin 3-gallate. Redox Biol. 2014;2:187-95.

37. Fraga CG, Oteiza PI, Galleano M. Plant bioactives and redox signaling: (-)-Epicatechin as a paradigm. Mol Asp Med. 2018;61:31-40.

38. Hassan FU, Rehman MS, Khan MS, Ali MA, Javed A, Nawaz A, et al. Curcumin as an alternative epigenetic modulator: mechanism of action and potential effects. Front Genet. 2019;4:514.

39. Cione E, La Torre C, Cannataro R, Caroleo MC, Plastina P, Gallelli L. Quercetin, epigallocatechin gallate, curcumin, and resveratrol: from dietary sources to human microRNA modulation. Molecules. 2019;25:63.

40. Oliveira AR, Cruz KJ, Severo JS, Morais JB, Freitas TE, Araújo RS, et al. Hypomagnesemia and its relation with chronic low-grade inflammation in obesity. Rev Assoc Med Bras. 2017;63:156-63.

41. Abdali D, Samson SE, Grover AK. How effective are antioxidant supplements in obesity and diabetes?. Med Princ Pract. 2015;24:201-15.

42. Zulet MA, Puchau B, Hermsdorff HH, Navarro C, Martínez JA. Dietary selenium intake is negatively associated with serum sialic acid and metabolic syndrome features in healthy young adults. Nutr Res. 2009;29:41-8.

43. Crilly MA, McNeill G. Arterial dysfunction in patients with rheumatoid arthritis and the consumption of daily fruits and daily vegetables. Eur J Clin Nutr. 2012;66:345-52.

44. Noad RL, Rooney C, McCall D, Young IS, McCance D, McKinley MC, et al. Beneficial effect of a polyphenol-rich diet on cardiovascular risk: a randomised control trial. Heart. 2016;102:1371-9. 
45. McCall DO, McGartland CP, McKinley MC, Patterson CC, Sharpe P, McCance DR, et al. Dietary intake of fruits and vegetables improves microvascular function in hypertensive subjects in a dose-dependent manner. Circulation. 2009;119:2153-60.

46. van Bussel BCT, Henry RMA, Schalkwijk CG, Dekker JM, Nijpels G, Feskens EJM, et al. Alcohol and red wine consumption, but not fruit, vegetables, fish or dairy products, are associated with less endothelial dysfunction and less low-grade inflammation: the Hoorn Study. Eur J Nutr. 2018;57:1409-19.

47. Akbari M, Tamtaji OR, Lankarani KB, Tabrizi R, Dadgostar E, Kolahdooz F, et al. The effects of resveratrol supplementation on endothelial function and blood pressures among patients with metabolic syndrome and related disorders: a systematic review and meta-analysis of randomized controlled trials. High Blood Press Cardiovasc Prev. 2019;26:305-19.

48. Hallajzadeh J, Milajerdi A, Kolahdooz F, Amirani E, Mirzaei H, Asemi Z. The effects of curcumin supplementation on endothelial function: a systematic review and meta-analysis of randomized controlled trials. Phytother Res. 2019;33:2989-95.

49. Yamagata K. Polyphenols regulate endothelial functions and reduce the risk of cardiovascular disease. Curr Pharm Des. 2019;25:2443-58.

50. Yamagata K, Yamori Y. Inhibition of endothelial dysfunction by dietary flavonoids and preventive effects against cardiovascular disease. J Cardiovasc Pharmacol. 2020;75:1-9.

51. Al Mheid I, Patel R, Murrow J, Morris A, Rahman A, Fike L, et al. Vitamin D status is associated with arterial stiffness and vascular dysfunction in healthy humans. J Am Coll Cardiol. 2011;58:186-92.

52. Giallauria F, Milaneschi $Y$, Tanaka T, Maggio M, Canepa M, Elango P, et al. Arterial stiffness and vitamin D levels: the Baltimore longitudinal study of aging. J Clin Endocrinol Metab. 2012;97:3717-23.

53. Mahmoud AM, Szczurek M, Hassan C, Masrur M, Gangemi A, Phillips SA. Vitamin $D$ improves nitric oxide-dependent vasodilation in adipose tissue arterioles from bariatric surgery patients. Nutrients. 2019;11:2521.

54. Dou D, Yang B, Gan H, Xie D, Lei H, Ye N. Vitamin D supplementation for the improvement of vascular function in patients with chronic kidney disease: a meta-analysis of randomized controlled trials. Int Urol Nephrol. 2019;51:851-8.

55. Gianfredi V, Bragazzi NL, Nucci D, Villarini M, Moretti M. Cardiovascular diseases and hard drinking waters: implications from a systematic review with metaanalysis of case-control studies. J Water Health. 2017;15:31-40.

56. Marques BCAA, Klein MRST, da Cunha MR, de Souza Mattos S, de Paula Nogueira $L$, de Paula $T$, et al. Effects of oral magnesium supplementation on vascular function: a systematic review and meta-analysis of randomized controlled trials. High Blood Press Cardiovasc Prev. 2020;27:19-28.

57. Connell P, Young VM, Toborek M, Cohen DA, Barve S, McClain CJ, et al. Zinc attenuates tumor necrosis factor-mediated activation of transcription factors in endothelial cells. J Am Coll Nutr. 1997;16:411-7.

58. Raij L, Nagy J, Coffee K, DeMaster EG. Hypercholesterolemia promotes endothelial dysfunction in vitamin E- and selenium-deficient rats. Hypertension. 1993;22:56-61.

59. Alehagen U, Aaseth J, Alexander J, Johansson P. Still reduced cardiovascular mortality 12 years after supplementation with selenium and coenzyme Q10 for four years: a validation of previous 10-year follow-up results of a prospective randomized doubleblind placebo-controlled trial in elderly. PLoS ONE. 2018;13:e0193120.

60. Kovács E, Siani A, Konstabel K, Hadjigeorgiou C, de Bourdeaudhuij I, Eiben G, et al. IDEFICS consortium. Adherence to the obesity-related lifestyle intervention targets in the IDEFICS study. Int J Obes. 2014;38:S144-51.
61. Van der Spuy WJ, Pretorius E. Is the use of resveretrol in the treatment and prevention of obesity premature? Nutr Res Rev. 2009;22:111-117.

62. Kocaadam B, Sanlier N. Curcumin, an active componenet of turmeric (Curcuma longa), and its effects on healthy. Crit Rev Food Sci Nutr. 2017;57:2889-95.

63. Carr A, Frei B. The role of natural antioxidants in preserving the biological activity of endothelium-derived nitric oxide. Free Radic Biol Med. 2000;28:1806-14.

\section{ACKNOWLEDGEMENTS}

We thank the Association Nati per Vivere (ANAVI) that donated Periflux 6000 System integrated with a thermostatic 457 probe, (Perimed), for the study.

\section{AUTHOR CONTRIBUTIONS}

$\mathrm{PA}, \mathrm{PL}, \mathrm{ZT}, \mathrm{AF}, \mathrm{RLA}$, and $\mathrm{PG}$ designed the research; $\mathrm{PA}, \mathrm{PL}, \mathrm{ZT}$, and $\mathrm{PC}$ conducted the research; PA, PL, ZT, and NF analyzed data; PA, PL, ZR, NF, PC, AF, RLA, and PG wrote the paper and had primary responsibility for final content.

\section{COMPETING INTERESTS}

The authors declare no competing interests.

\section{ADDITIONAL INFORMATION}

Correspondence and requests for materials should be addressed to Angelo Pietrobelli.

Reprints and permission information is available at http://www.nature.com/ reprints

Publisher's note Springer Nature remains neutral with regard to jurisdictional claims in published maps and institutional affiliations.

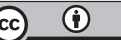

Open Access This article is licensed under a Creative Commons Attribution 4.0 International License, which permits use, sharing, adaptation, distribution and reproduction in any medium or format, as long as you give appropriate credit to the original author(s) and the source, provide a link to the Creative Commons license, and indicate if changes were made. The images or other third party material in this article are included in the article's Creative Commons license, unless indicated otherwise in a credit line to the material. If material is not included in the article's Creative Commons license and your intended use is not permitted by statutory regulation or exceeds the permitted use, you will need to obtain permission directly from the copyright holder. To view a copy of this license, visit http://creativecommons. org/licenses/by/4.0/.

(c) The Author(s) 2022, corrected publication 2022 